\title{
JOINT INFLUENCE OF STEERED VACUUM ARC AND NEGATIVE REPETITIVELY PULSED BIAS ON TITANIUM MACROPARTICLES SUPPRESSION
}

\author{
ALEXANDER RYABCHIKOV, PETER ANANIN, SERGEI DEKTYAREV, \\ ALEXEY SHEVELEV AND DENIS SIVIN \\ National research Tomsk Polytechnic University, Russia \\ ralex@tpu.ru
}

This paper presents the results of experimental study of titanium macroparticles accumulation on a negatively biased substrate immersed in DC vacuum arc plasma. Macroparticle and plasma emission properties of random and steered arc evaporators were investigated. It was shown that using of steered arc with a tangential magnetic field strength 200 Gs reduces the generation of macroparticles 4 fold compared to a random arc source. Application of repetitively pulsed negative bias significantly decreases macroparticle assembling on a substrate surface for both evaporator designs. After 20 minutes of ion-plasma treatment with negatively pulsed bias $(-2 \mathrm{kV}, 7 \mu \mathrm{s}$, 105 p.p.s.) and steered arc, the observed macroparticle surface density appears 2 orders of magnitude smaller than after vacuum arc plasma deposition at anode potential with random arc. Thus, the possibility of high-frequency short-pulse plasma immersion ion implantation by implementing DC vacuum arc plasma is discussed.

Keywords: Vacuum steered arc, Plasma, Macroparticles, High frequency pulsed bias. 